\title{
Research on Automatic Steering Control System for Plant Protection Robots
}

\author{
Wei Zhang ${ }^{1}$, Chang-sheng $\mathrm{Ai}^{1,{ }^{*}}$, De-lin $\mathrm{Wu}^{2}$,Wei-xin $\mathrm{Li}^{2}$ and Yong-ze Zhang ${ }^{1}$ \\ ${ }^{1}$ School of Mechanical Engineering, University of Jinan, Jinan 250022, China \\ ${ }^{2}$ Shandong ZhongHe Plant Protection Machinery Ltd,Yantai 265300,China \\ ^Email:me_aics@ujn.edu.cn
}

\begin{abstract}
Keywords: plant protection robot, deviation control strategy, unmanned, automatic control
Abstract. Aiming at the problem of agricultural robots such as being difficult to adapt to the complex operating environment in field ,highter cost for promotion and application ,designed an automatic steering system based on the steering wheel angle-sensor and the steering motor ,formed a closed-loop control system by sensor and motor .Taking STM32F103 single chip microcomputer as the core of the control system, the PID control algorithm based on the model of steering deviation , and analyzed and verified the effectiveness of the steering drive. The experimental results show that the maximum mean absolute error of the steering wheel angle is 0.60 degrees, and the angle of the target is obtained by $0.8-1.0 \mathrm{~s}$.
\end{abstract}

\section{Introduction}

The robot is the inevitable choice that to realize the agricultural production toward high efficiency and high accuracy of mechanization (informatization and intelligent ), robots can reduce the labor strength, improve the convenience and comfort of the workers, at the same time to ensure the safety of the people, reflect the people-oriented ${ }^{[1]}$. Therefore, how to further improve the application level of agricultural robots, so as to adapt to the requirements of the complex farmland environment, has become the focus of research at home and abroad ${ }^{[2]}$.

Min $\mathrm{H} \mathrm{K}^{[3]}$ discusses the design process of the omni-directional mobile robot, used genetic algorithm to optimize the steering control system of the robot; Zhang Tiemin ${ }^{[4]}$ researched the ability that agricultural wheeled mobile car in different load conditions, and analyzes each driving motor torque distribution. But the current domestic research that oriented agricultural robot is mainly focused on differential steering control method ${ }^{[5]}$, but the driving wheel will skid in the harsh environment of farmland, due to steering effect is not up to the requirements of the job.

According to the actual requirements that complex environment and operation of plant protection , designed an automatic steering system based on the steering wheel angle-sensor and the steering motor ,formed a closed-loop servo control system by sensor and motor , and according to the modular design method to construct the hardware and software of the control system, so that the system could realize the veer task.

\section{The working principle of automatic steering control system}

The target angle $\alpha_{0}$ that from upper computer or the remote controller compared with the actual angle $\alpha_{i}$, could got deviation named $\Delta \alpha$, deviation is transmitted to the PID controller as the input value, and PID export the required value according to positive and negative deviation, when the deviation is positive, the steering motor is transferred, and inversion; when the deviation is large, controller output large duty cycle PWM (pulse width modulation), in addition, the steering wheel angle sensor will real-time detection, the angle got will contrast with deviation, when the deviation is equal to 0 , to complete the task of steering. The steering wheel sensor is used as the measuring device, the steering motor is an actuator, and the single chip microcomputer is the control system. Figure 1 for the working principle of automatic steering control system. 


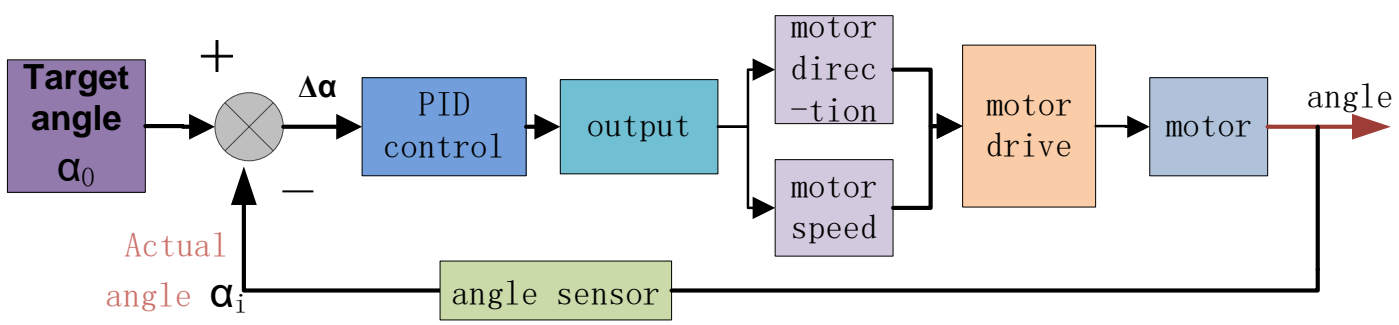

Figure 1 the working principle of automatic steering control system

\section{The hardware automatic steering control system}

The robot steering system used in the field of plant protection especially in complex operation ,according to the requirements of the rapid response,designed automatic steering system. the control system is mainly composed of a central processor module (STM32 MCU), terminal module (steering actuator motor), steering wheel angle detection module. Figure 2 is the general body system. Figure 2 is the characters of robot automatic steering control system hardware composition diagram.

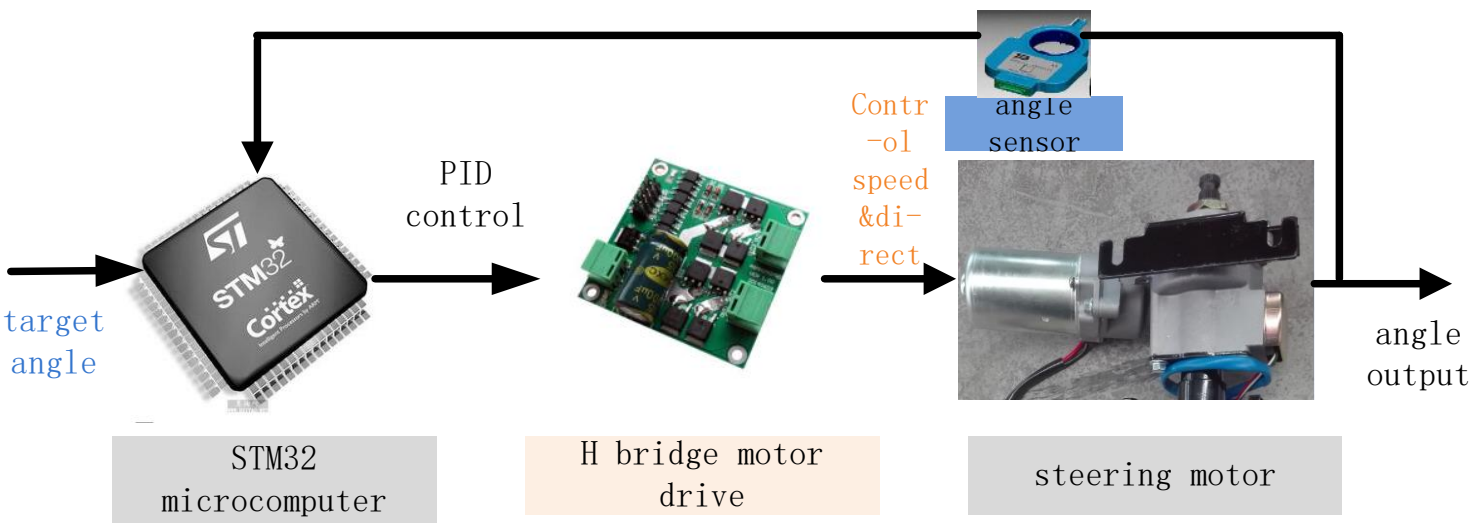

Figure 2 characters of robot automatic steering control system hardware composition diagram

\section{Steering wheel Angle sensor module design}

Steering wheel Angle sensor is the key to realize the closed loop control, its main function is to $\mathrm{m}$ easure the string around the point of view, and output the Angle signal to the MCU as a closed loop control of the input signal ${ }^{[6]}$. Angle sensor has main hole, when connecting to the steering column shaft, 1/16 ring spindle each roll, sensor technology to increase 1 . When the motor shaft rotates in on e direction, the count increase, on the other hand, the count to reduce ${ }^{[7]}$. Table 1 for testing paramet ers of angle sensor.

Table 1 testing parameters of angle sensor

\begin{tabular}{ll}
\hline major parameter & parameter standard \\
\hline range & $\pm 900^{\circ}$ \\
resolution & $\pm 0.01^{\circ}$ \\
temperature range & -40 to $+85^{\circ} \mathrm{C}$ \\
firmware upgrade & CAN Bus 2.0 \\
\hline
\end{tabular}

Input angle $\alpha_{0}$ as a closed loop control target, steering wheel angle sensor to get the absolute angle in the process of robot walking and with MCU angle formed closed-loop control,the sensor is the way that uses CAN bus and MCU data receiving and sending data ${ }^{[8]}$, the sensor send 8 bytes, among them, the first and the second byte is the collected information. Figure 4 for the turning angle of steering wheel Angle sensor collected information.

\section{Actuator module design}

Power steering motor is the module of the robot steering system, responsible for the completion of 
the steering task, is the action part of the entire system , the steering motor connecting rack and pinion steering machine, through the acceptance of isolated DC motor drive module AQMH2403ND driver $^{[9]}$,realization of $\mathrm{H}$ bridge using $\mathrm{P}$ and $\mathrm{N}$ complementary MOS tube .

When the deviation occurs, the single chip microcomputer control drive motor module IN1 and IN2, simultaneously control the ENA pin output duty cycle of different PWM square wave, realize the control of the steering motor, complete the robot's steering task.Table 2 is signal logic for the motor drive module.

Table 2 signal logic for the motor drive module

\begin{tabular}{cccc}
\hline IN1 & IN2 & ENA & OUT1、OUT2 \\
\hline 0 & 0 & $\times$ & brake \\
1 & 1 & $\times$ & dangling \\
1 & 0 & PWM & $\begin{array}{c}\text { forward speed } \\
\text { regulation } \\
\text { reversal speed } \\
\text { regulation }\end{array}$ \\
0 & 1 & PWM & full forward speed \\
1 & 0 & 1 & full reversal speed \\
\hline
\end{tabular}

\section{The central processor module (STM32 microcontroller)}

The robot steering system uses the stmicroelectronics production series of STM32F103 microcontroller as the main work, choose the main reason of the single chip microcomputer has 3 points: 1) it contains an enhanced CAN bus communication module, the support of the highest 1 $\mathrm{MB} / \mathrm{s}$ data transfer rate, and has eight send or receive buffer register; 2) equipped with a serial communication interface, enhanced with a remote control or PC to keep data transmission function; 3 ) has a multi-channel PWM output pin, which can realize the 8-bit resolution of duty ratio adjustment function, so as to achieve the goal of control motor speed. Figure 3 for microcontroller pins and CAN send and receive.
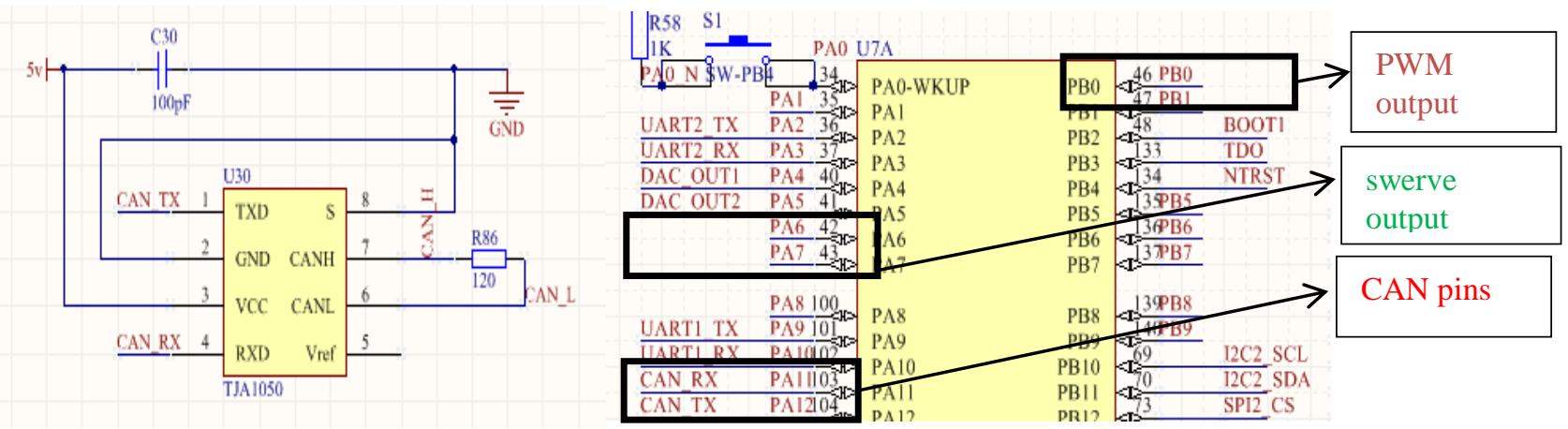

Figure 3 microcontroller pins and CAN send and receive

\section{The software of automatic steering control system}

After power on reset, the system is initialized, the initialization process mainly includes: CAN bus initialization, MCU I/O port, motion control program and PID control program initialization. After the initialization of MCU, the target angle, and comparing with the actual angle deviation, the PID control system according to the deviation value, output speed signal to the DC motor, and drive the steering motor to achieve to change direction. Figure 4 is the flow chart of robot automatic steering control system . 


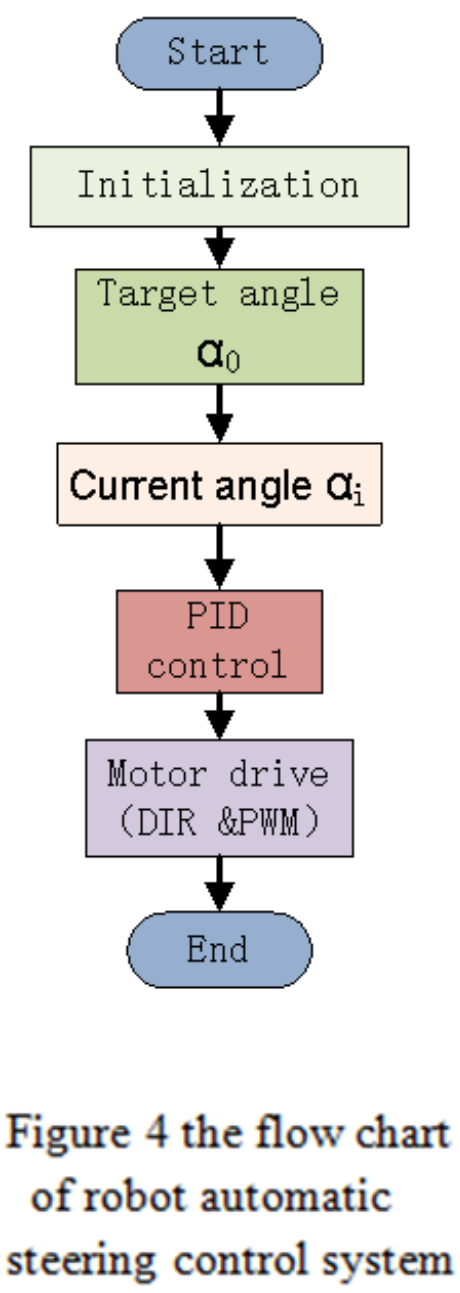

\section{Conclusion}

In this paper, based on the power steering motor and steering wheel angle sensor closed-loop servo control system, complete automatic steering task robot operations, also takes STM32F103 microcontroller as the core of control system design, has a simple design, the advantages of flexible and low cost, the maximum average absolute error to control the angle is 0.60 degrees, the control effect is strong ,after 0.8-1.0s get the target angle, achieve stability especially suitable for orchard vineyard crop growth period of plant operation, it has a broad application prospects.

\section{Acknowledgement}

This work was financially supported by the Shandong Province Key Projects, The Self-propelled Robots Based on Network Visual Feedback and Telecontrolled for Plant Protection,The Plan of Shandong Province Agricultural Equipment Research and Development Innovation,[2015]9.

\section{References}

[1] Lin Huan, $\mathrm{Xu}$ Linyun, The development and prospect of agricultural robots in China[J]. Acta Agriculturae Zhejiangensis,2015,27(5):865-871.

[2] Ji Jiangtao, Zheng Zhihua, Current Situation and Development Tendency of Agriculture Robot[J]. Agricultural Mechanization Research,2014,36(2):1-4. 
[3]Min $\mathrm{H} \mathrm{K}$,et al. Autonomous greenhouse mobile robot driving strategiesfrom system integration perspective: Review and application[C]//Transactions on Mechatronics. America: IEEE /ASME, 2014:1-12.

[4] Zhang Tiemin,Huang Han, Design and test of drive and control system for electric wheeled mobile car. Transactions of Chinese Society of Agricultural Engineering ,2014,30(9).

[5]Shuai Zhibin, Zhang Hui, et al. Lateral motion-control for four-wheel-independent-drive electric vehicles-using optimal torque allocation and dynamic message priortyscheduling $[\mathrm{J}]$. Control Engi -neering Practice, 2014, 3(1): 55-66.

[6] Zhang Yutao, The development of automotive angular position sensor assembly test bench[D]., Harbin Institute of Technology,2013.

[7] Li Hao,Xu Yanliang,et al. Study on the steering wheel angle sensor used for electric vehicle[J]. Transducer and Microsystem Technologies,2011,30(3).

[8]Ding E L, Fennel H, Ding S X. Model-based diagnosis of sensor faults for ESP systems[J]. Contr ol engineering practice, 2004, 12(7): 847-856.

[9]Kim J H, Song J B. Control logic for an electric power steering system using assist motor[J]. Mechatronics, 2002, 12(3): 447-459. 